\title{
Clinical characteristics, inflammation and coagulation status in patients with immunological disease-related chronic cerebrospinal venous insufficiency
}

\author{
Si-Ying Song ${ }^{1,2,3}$, Duo Lan ${ }^{1,2,3}$, Xiao-Qin Wu ${ }^{1,2,3}$, Yu-Chuan Ding ${ }^{2,3,4}$, Xun-Ming Ji ${ }^{1,2,3}$, Ran Meng ${ }^{1,2,3}$ \\ ${ }^{1}$ Department of Neurology, Xuanwu Hospital, Capital Medical University, Beijing, China; ${ }^{2}$ Advanced Center of Stroke, Beijing Institute for Brain \\ Disorders, Beijing, China; ${ }^{3}$ Department of China-America Institute of Neuroscience, Xuanwu Hospital, Capital Medical University, Beijing, China; \\ ${ }^{4}$ Department of Neurosurgery, Wayne State University School of Medicine, Detroit, Michigan, USA \\ Contributions: (I) Conception and design: SY Song, R Meng; (II) Administrative support: SY Song, R Meng; (III) Provision of study materials or \\ patients: SY Song, R Meng; (IV) Collection and assembly of data: SY Song, D Lan, XQ Wu; (V) Data analysis and interpretation: SY Song, R Meng, \\ XM Ji, YC Ding; (VI) Manuscript writing: All authors; (VII) Final approval of manuscript: All authors. \\ Correspondence to: Ran Meng, MD, PhD. Department of Neurology, Xuanwu Hospital, Capital Medical University, Chang Chun Road 45, Xicheng, \\ Beijing, China. Email: ranmeng2011@pku.org.cn.
}

Background: Immunological disease-related chronic cerebrospinal venous insufficiency (CCSVI) is rarely reported. This study aimed to analyze clinical characteristics, inflammation, and coagulation status in patients with immunological disease-related CCSVI.

Methods: Patients with CCSVI were enrolled from 2017 to 2019 and divided into three cohorts based on their immunological disease backgrounds, including groups with confirmed autoimmune disease, with suspected/subclinical autoimmune disease, and with non-immunological etiology. Immunological, inflammatory, and thrombophilia biomarker assay in blood samples were obtained. Mann-Whitney $U$ test or Fisher's exact test was used to compare continuous variables or categorical variables between the CCSVI patients with or without the immunological etiology. Spearman's correlation analysis was conducted among age, baseline neutrophil-to-lymphocyte ratio (NLR), platelet-to-lymphocyte ratio (PLR), interleukin-6 (IL-6), C-reactive protein (CRP), and neuron-specific enolase (NSE) in the three groups.

Results: A total of 255 consecutive patients with CCSVI were enrolled, including three subgroups: CCSVI with confirmed autoimmune disease $(n=41)$, CCSVI with suspected/subclinical autoimmune disease $(n=116)$ and CCSVI with non-immunological etiology ( $n=98)$. In the first subgroup, a series of 41 cases was confirmed with eight different autoimmune diseases including antiphospholipid syndrome ( $n=18)$, Sjögren's syndrome $(\mathrm{n}=8)$, immunoglobulin G4-related disease $(\mathrm{n}=7)$, Behçet's disease $(\mathrm{n}=2)$, autoimmune hepatitis $(\mathrm{n}=2)$, Wegener's granulomatosis $(n=2)$, systemic sclerosis $(n=1)$ and AQP4 antibody-positive neuromyelitis optica spectrum disorder $(\mathrm{n}=1)$. Groups with immunological etiology did not show a higher incidence of thrombophilia or increased proinflammatory biomarkers (e.g., neutrophil, IL-6). However, patients with non-immunological etiology had a higher baseline level of CRP. Additionally, baseline PLR was moderately correlated to NLR and CRP in CCSVI patients with non-immunological etiology and suspected/subclinical autoimmune disease.

Conclusions: The formation of CCSVI may be based on the inflammatory process, facilitated by multiple risk factors, among which medical history of immunological diseases may play a significant role due to the intricate relationship between inflammation and coagulation. Moreover, CCSVI may also cause an independent inflammatory injury in venous walls, leading to focal stenosis or thrombus, without attacks from autoimmune antibodies.

Keywords: Chronic cerebrospinal venous insufficiency (CCSVI); cerebral venous sinus stenosis (CVSS); internal jugular vein stenosis (IJVS); inflammatory biomarkers; autoimmune disease

Submitted May 23, 2020. Accepted for publication Nov 13, 2020.

doi: 10.21037/atm-20-4201

View this article at: http://dx.doi.org/10.21037/atm-20-4201

(C) Annals of Translational Medicine. All rights reserved. 


\section{Introduction}

Chronic cerebrospinal venous insufficiency (CCSVI), as a state of impaired intracranial or extracranial venous drainage, has been heatedly discussed over its role in the pathogenesis of multiple sclerosis (MS) in the last decades (1-4). However, with the increasing evidence of CCSVI not unique to MS (5-7), the relationship between CCSVI and other autoimmune diseases has emerged. Given that autoimmune diseases are associated with hypercoagulation state due to elevated autoimmune antibodies, venous thromboembolism (VTE) is one of the most common complications. Nevertheless, studies on autoimmune disease-mediated cerebral venous sinus thrombosis (CVST) are rather rare. Only a few cohort studies of patients with systemic lupus erythematosus (SLE) (8-11) or Behçet's disease (BD) $(12,13)$ were reported to have CCSVI as well. Moreover, several case series presented patients with the antiphospholipid-antibody syndrome (APS) $(14,15)$, inflammatory bowel disease (IBD) (16), or Wegener's granulomatosis $(17,18)$ with the coexistence of CVST.

The clinical features of autoimmune disease-mediated CCSVI are still unclear. Moreover, we also found a number of patients with CCSVI had positive tests of immunological biomarkers, such as decreased complement 3 (C3) or complement 4 (C4), increased erythrocyte sedimentation rate (ESR), immunoglobulin $\mathrm{G}$ ( $\mathrm{IgG}$ ), or immunoglobulin $\mathrm{E}(\mathrm{IgE})$, despite negative findings of antinuclear bodies or antineutrophil cytoplasmic antibodies. Thus, we aimed to enroll CCSVI patients with confirmed autoimmune disease or suspected/subclinical autoimmune disease and provided comprehensive clinical features of CCSVI with or without immunological etiology. Furthermore, thanks to the intricate relationship between coagulation, inflammation (adaptive immune system), and complement pathway (innate immune system), we evaluated relevant biomarkers in this study. The following article is presented in accordance with the STROBE reporting checklist (available at http://dx.doi. org/10.21037/atm-20-4201).

\section{Methods}

\section{Population}

A total of 255 consecutive patients with confirmed CCSVI were enrolled, with admission in the Department of Neurology, Xuanwu Hospital, Capital Medical University, from 2017 to 2019. The study was conducted in accordance with the Declaration of Helsinki (as revised in 2013). The study was approved by Ethics Committee of Xuanwu Hospital, Capital Medical University (2019-006), and informed consent was taken from all individual participants.

Inclusion criteria were as follows: (I) patients with CCSVI, including internal jugular vein stenosis (IJVS), cerebral venous sinus stenosis (CVSS), or CVSS combined with IJVS were confirmed by contrast-enhanced magnetic resonance venography (CE-MRV) or digital subtraction angiography (DSA); (II) patients did not have parenchymal lesions due to CCSVI; (III) the course of the disease was at a subacute or chronic stage, defined as an interval of more than 4 weeks; (IV) patients with autoimmune diseases were confirmed by the Department of Rheumatology, Xuanwu Hospital, Capital Medical University.

We excluded patients with definite acute or chronic infection; use of anti-inflammatory medication within 4 weeks prior to blood collection; intracranial hypertension (IH) resulting from other reasons: (I) drug-induced $\mathrm{IH}$; (II) cerebrospinal fluid shunt history; (III) intracranial mass occupation; (IV) arteriovenous malformations.

\section{Clinical and demographic data}

Age, gender, course of the disease (from onset to admission), treatments, and presumable risk factors known before hospitalization or discovered during hospitalization were recorded. The common risk factors included hypertension (use of antihypertensive medications or systolic blood pressure $>140 \mathrm{mmHg}$ or diastolic blood pressure $>90 \mathrm{mmHg}$ before hospitalization diastolic blood pressure $>90 \mathrm{mmHg}$ before hospitalization), diabetes mellitus (use of antidiabetic therapies or fasting blood glucose $>7 \mathrm{mmol} / \mathrm{L}$ on two occasions during hospitalization), hypercholesterolemia (use of lipid-lowering medications or low-density lipoprotein cholesterol $>1 \mathrm{~g} / \mathrm{L}$ ), a history of myocardial infarction or angina, overweight (body mass index $>25 \mathrm{~kg} / \mathrm{m}^{2}$ ), anemia (hemoglobin $<12.5 \mathrm{~g} / \mathrm{dL}$ ), hepatitis B virus (HBV) infection (use of anti-HBV therapies or positive hepatitis B core antibody/antigen or hepatitis B e antibody/antigen), hyperhomocysteinemia ( $>15 \mathrm{mmol} / \mathrm{L})$, hyperuricemia ( $>416 \mu \mathrm{mol} / \mathrm{L}$ ), chronic rhinosinusitis, history of otitis media/ mastoiditis, suspected thyroid disorders (including either abnormal thyroid ultrasound results or abnormal thyroid function results), autoimmune disease, thrombophilia (including protein $\mathrm{S}$ deficiency, protein $\mathrm{C}$ deficiency, antithrombin-III deficiency, hyperfibrinogenemia, primary thrombocythemia or increased D-dimer level), and history of ischemic or hemorrhagic stroke. We also collected 
clinical signs, such as papilledema and IH. The severity of papilledema was evaluated by the Frisen papilledema grade criteria. Intracranial pressure (ICP) was detected by lumbar puncture, and IH was defined as ICP more than $200 \mathrm{mmH}_{2} \mathrm{O}$.

Subgroup analysis was conducted based on etiology. CCSVI patients with immunological etiology were defined as the coexistence of confirmed autoimmune disease or suspected/subclinical autoimmune disease, while CCSVI patients with non-immunological etiology included CVSTrelated CCSVI or bone/vessel/lymph node-compressionrelated CCSVI.

\section{Immunological, inflammatory, and thrombophilia biomarkers assay in the blood sample}

Immunological biomarker assay included autoimmune antibody tests in serum samples, including antinuclear antibodies (ANAs), anti-neutrophil cytoplasmic antibodies (ANCAs), and antiphospholipid antibodies (APLAs), as well as other immunological markers, such as $\mathrm{C} 3, \mathrm{C} 4, \mathrm{IgG}, \mathrm{IgE}$, ESR, and rheumatic factor (RF).

Inflammatory biomarker assay consisted of neutrophilto-lymphocyte ratio (NLR) and platelet-to-lymphocyte ratio (PLR) in plasma EDTA samples, as well as interleukin-6 (IL-6), C-reactive protein (CRP), and neuron-specific enolase (NSE) in serum samples. Baseline levels were measured on admission. NLR was computed using the absolute neutrophil count divided by the absolute lymphocyte count. PLR was calculated using the absolute platelet count divided by the absolute lymphocyte count. Baseline inflammatory markers were considered as continuous variables.

Thrombophilia biomarker assay evaluated both antigens, including platelet, fibrinogen, d-dimer, antithrombinIII, protein $\mathrm{C}$, and protein $\mathrm{S}$, as well as activity, such as thrombin time, partial thromboplastin time (PTT) and activated PTT (aPTT) in plasma sodium-citrate sample without platelet depleted. Cutoff values were based on the referential interval in the Laboratory of Xuanwu Hospital, Capital Medical University.

All blood samples were collected in VACUETTE ${ }^{\circledR}$ Blood Collection Tubes (Greiner Bio-One, Kremsmünster, Austria). Detailed information on coagulation and inflammatory kits and instruments is presented in Table S1.

\section{Statistical analysis}

Bartlett's test for equal variances and the Shapiro-Wilk normality test for distribution were conducted for each continuous variable. We then used the Mann-Whitney $U$ test or Fisher's exact test to compare continuous variables or categorical variables between patients with immunological etiology CCSVI and non-immunological etiology CCSVI. Difference between levels of baseline inflammatory markers [NLR, PLR, and red blood cell distribution width (RDW)] and that at discharge was tested by Wilcoxon signedrank test. Correlation coefficients were calculated with Spearman's test among age, baseline NLR, PLR, RDW, IL-6, CRP, and NSE. Quantitative variables with a normal distribution were specified as mean \pm standard deviation, and those with abnormal distribution were expressed as median with interquartile range (IQR). Differences were considered significant at a two-sided $\mathrm{P}<0.05$ level. Analyses were performed with Stata software (version 15.0 SE, Stata Corp., LP, Texas, USA) and R software [version 3.6.2 (2019-12-12)].

\section{Results}

\section{Baseline clinical features}

A total of 255 patients (104 males and 151 females) with CCSVI were enrolled in this retrospective study, of which more than $95 \%$ had a disease course over 1 month (chronic stage), and followed up with $18.13 \pm 5.58$ months. Patients most likely presented with sleep disturbances (60.4\%), eye discomfort $(58.4 \%)$, head noise $(53.7 \%)$, tinnitus $(51.8 \%)$, headache $(45.1 \%)$, and hearing loss $(32.2 \%)$. Combined risk factors were commonly seen, for instance, thrombophilia state, overweight, hyperlipidemia, hypertension, anemia, and suspected thyroid disorders. Based on locations of CCSVI, IJVS, CVSS, and CVSS combined with IJVS were found in $68.2 \%, 16.9 \%$, and $14.9 \%$ of enrolled patients, respectively. Treatments for CCSVI patients were antiplatelet drugs $(60.0 \%)$, anticoagulants $(32.2 \%)$, and endovascular therapy $(12.5 \%)$. Details were displayed in Table 1.

\section{Difference between CCSVI with or without immunological etiology}

Immunological disease-related CCSVI was defined as confirmed autoimmune disease or suspected/subclinical autoimmune disease. The prevalence of autoimmune disease-related CCSVI was relatively low as $16.1 \%(n=41)$, while CCSVI was rather common in suspected/subclinical autoimmune disease $(45.5 \%, \mathrm{n}=116)$. A total of 41 cases 
Table 1 Demographic and basic clinical features

\begin{tabular}{|c|c|c|c|c|c|}
\hline Variables & All $(n=255)$ & \multicolumn{2}{|c|}{ Immunological etiology $(n=157)$} & $\begin{array}{l}\text { Non-immunological } \\
\text { etiology }(n=98)\end{array}$ & $P$ value \\
\hline Age, years & $53.47 \pm 15.04$ & $50.88 \pm 18.77$ & $56.42 \pm 14.14^{*}$ & $51.05 \pm 13.81$ & 0.017 \\
\hline Gender (M:F) & 104:151 & $14: 27$ & $54: 62$ & $36: 62$ & 0.223 \\
\hline Subacute (within 1 month) & $11(4.3)$ & $3(7.3)$ & $4(3.4)$ & $4(4.1)$ & \\
\hline Chronic (more than 1 month) & $244(95.7)$ & $38(92.7)$ & $112(96.6)$ & $94(95.9)$ & \\
\hline Follow-up time, months ${ }^{\wedge}$ & $18.13 \pm 5.58$ & $18.27 \pm 5.52$ & $18.46 \pm 5.31$ & $17.69 \pm 5.94$ & 0.656 \\
\hline mRS on admission & $0.43 \pm 0.68$ & $0.60 \pm 0.60$ & $0.42 \pm 0.61$ & $0.39 \pm 0.77$ & 0.110 \\
\hline Papilledema & $49(19.2)$ & $16(39.0)$ & $17(14.7)$ & $16(16.3)$ & 0.004 \\
\hline Frisen scale & $1.08 \pm 1.31$ & $1.56 \pm 1.47$ & $0.82 \pm 1.23$ & $1.03 \pm 1.21$ & 0.139 \\
\hline Head noises & $137(53.7)$ & $18(43.9)$ & $72(62.1)$ & $47(48.0)$ & 0.045 \\
\hline Tinnitus & $132(51.8)$ & $21(51.2)$ & $61(52.6)$ & $50(51.0)$ & 0.985 \\
\hline Headache & $115(45.1)$ & $20(48.8)$ & $43(37.1)$ & $52(53.1)$ & 0.055 \\
\hline Neck discomfort & 77 (30.2) & $9(22.0)$ & $42(36.2)$ & $26(26.5)$ & 0.152 \\
\hline Hearing loss & $82(32.2)$ & $11(26.8)$ & 39 (33.6) & $32(32.7)$ & 0.738 \\
\hline Type 2 diabetes mellitus & $22(8.6)$ & $4(9.8)$ & $11(9.5)$ & $7(7.1)$ & 0.794 \\
\hline HBP & $83(32.5)$ & $15(36.6)$ & $35(30.2)$ & $33(33.7)$ & 0.727 \\
\hline Hyperlipidemia & $90(35.3)$ & $11(26.8)$ & $47(40.5)$ & $32(32.7)$ & 0.236 \\
\hline Anemia & $57(22.4)$ & $13(31.7)$ & $26(22.4)$ & $18(18.4)$ & 0.234 \\
\hline Stroke & $20(7.8)$ & $2(4.9)$ & $10(8.6)$ & $8(8.2)$ & 0.821 \\
\hline Hemorrhage & $6(2.4)$ & $0(0.0)$ & $3(2.6)$ & $3(3.1)$ & 0.747 \\
\hline Hyperuricemia & $18(7.1)$ & $5(12.2)$ & $4(3.4)$ & $9(9.2)$ & 0.075 \\
\hline Hyperhomocysteinemia & $20(7.8)$ & 7 (17.1) & $7(6.0)$ & $6(6.1)$ & 0.082 \\
\hline CAD & $27(10.6)$ & $7(17.1)$ & $12(10.3)$ & $8(8.2)$ & 0.264 \\
\hline Previous otitis media/mastoiditis & $7(2.7)$ & $3(7.3)$ & $2(1.7)$ & $2(2.0)$ & 0.175 \\
\hline Chronic rhinosinusitis & $14(5.5)$ & $4(9.8)$ & $6(5.2)$ & $4(4.1)$ & 0.431 \\
\hline
\end{tabular}

Table 1 (continued) 
Table 1 (continued)

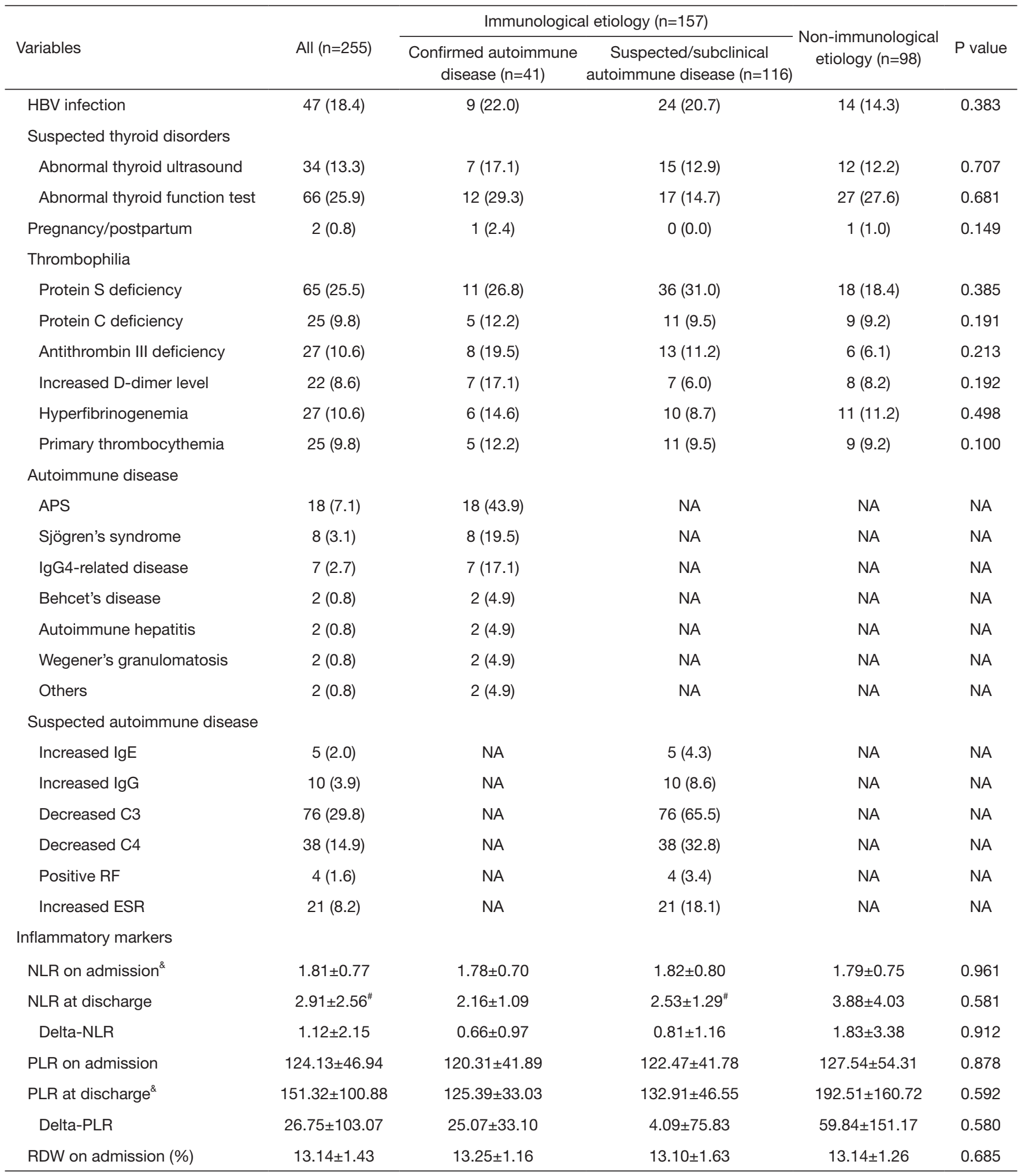

Table 1 (continued) 
Table 1 (continued)

\begin{tabular}{|c|c|c|c|c|c|}
\hline \multirow[b]{2}{*}{ Variables } & \multirow[b]{2}{*}{ All $(n=255)$} & \multicolumn{2}{|c|}{ Immunological etiology $(n=157)$} & \multirow[b]{2}{*}{$\begin{array}{l}\text { Non-immunological } \\
\text { etiology }(n=98)\end{array}$} & \multirow[b]{2}{*}{$P$ value } \\
\hline & & $\begin{array}{l}\text { Confirmed autoimmune } \\
\text { disease }(n=41)\end{array}$ & $\begin{array}{c}\text { Suspected/subclinical } \\
\text { autoimmune disease }(n=116)\end{array}$ & & \\
\hline Delta-RDW (\%) & $0.44 \pm 2.37$ & $0.28 \pm 0.95$ & $0.42 \pm 2.09$ & $0.95 \pm 3.30$ & 0.462 \\
\hline IL-6 (pg/mL) & $4.70 \pm 5.71$ & $6.36 \pm 7.51$ & $4.65 \pm 5.91$ & $4.12 \pm 4.48$ & 0.391 \\
\hline NSE (ng/mL) & $12.95 \pm 2.72$ & $13.56 \pm 3.33$ & $12.82 \pm 2.26$ & $12.88 \pm 2.95$ & 0.734 \\
\hline Localization of CVSS/IJVS & & & & & 0.026 \\
\hline CVSS & $43(16.9)$ & $7(17.1)$ & $13(11.2)$ & $23(23.5)$ & \\
\hline SSS & $15(5.9)$ & $2(4.9)$ & $5(4.3)$ & $8(8.2)$ & 0.523 \\
\hline LSigS & $22(8.6)$ & $6(14.6)$ & $5(4.3)$ & $11(11.2)$ & 0.058 \\
\hline RSigS & $19(7.5)$ & 8 (19.5) & $5(4.3)$ & $6(6.1)$ & 0.011 \\
\hline IJVS & $174(68.2)$ & $24(58.5)$ & $90(77.6)$ & $60(61.2)$ & \\
\hline LIJV-J1 segment & $16(6.3)$ & $5(12.2)$ & $7(6.0)$ & $4(4.1)$ & 0.218 \\
\hline RIJV-J1 segment & $11(4.3)$ & $4(9.8)$ & $4(3.4)$ & $3(3.1)$ & 0.195 \\
\hline LIJV-J2 segment & $26(10.2)$ & $5(12.2)$ & $13(11.2)$ & $8(8.2)$ & 0.667 \\
\hline RIJV-J2 segment & $12(4.7)$ & $2(4.9)$ & $5(4.3)$ & $5(5.1)$ & 1.000 \\
\hline LIJV-J3 segment & $144(56.5)$ & $21(51.2)$ & $71(61.2)$ & $52(53.1)$ & 0.530 \\
\hline Endovascular therapies & $32(12.5)$ & $8(19.5)$ & $10(8.6)$ & $14(14.3)$ & 0.138 \\
\hline Stenting & $25(9.8)$ & 8 (19.5) & $9(7.8)$ & $8(8.2)$ & 0.096 \\
\hline Balloon dilation & $5(2.0)$ & $0(0.0)$ & $2(1.7)$ & $3(3.1)$ & 0.592 \\
\hline Intrasinus thrombolysis & $4(1.6)$ & $0(0.0)$ & $0(0.0)$ & $4(4.1)$ & 0.035 \\
\hline ONSD & $8(3.1)$ & $4(9.8)$ & $1(0.9)$ & $3(3.1)$ & 0.026 \\
\hline
\end{tabular}

Data were presented as mean \pm standard deviation or $\mathrm{n}(\%) .{ }^{*}$, compared with group of non-immunological etiology group, statistically significant at $\mathrm{P}<0.05 ;{ }^{*}$, the number of patients who had complete blood count $(C B C)$ test at discharge $(n=36) ; \wedge$, time from discharge to follow-up (months); ", compared with group of NLR tested on admission, statistically significant at $P<0.05$. $P=0.001$ in general groups $(n=255), P=0.008$ in group with suspected/subclinical autoimmune disease $(n=116)$. mRS, modified Rankin scale; HBP, high blood pressure; CAD, coronary artery disease; HBV, hepatic type B virus; APS, antiphospholipid syndrome; C3, complement 3; C4, complement 4; RF, rheumatic factor; ESR, erythrocyte sedimentation rate; NLR, neutrophil to lymphocyte ratio; PLR, platelet to lymphocyte ratio; RDW, red blood cell distribution width; IL-6, interleukin-6; CRP, C-reactive protein; NSE, Neuron-specific enolase; CVSS, cerebral venous sinus stenosis; IJVS, internal jugular vein stenosis; SSS, superior sagittal sinus; TS, transverse sinus; LTS, left transverse sinus; RTS, right transverse sinus; SigS, sigmoid sinus; LSigS, left sigmoid sinus; RSigS, right sigmoid sinus; ONSD, optic nerve sheath decompression; NA, not applicable. 
with confirmed autoimmune disease-related CCSVI (14 males and 27 females, mean age $50.88 \pm 18.77$ years) were collected, among which, APS ( $\mathrm{n}=18$ ), Sjögren's syndrome (SS) $(\mathrm{n}=8)$, and IgG4-related disease (IgG4-RD) $(\mathrm{n}=7)$ were highly prevalent in our cohort. Apart from specific elevated autoantibodies, such as ANAs or ANCAs or APLAs, abnormal levels of nonspecific immunological markers were also coexisted within the CCSVI cohort of confirmed autoimmune disease, for instance, a decreased level of C3 $(n=16)$ or $\mathrm{C} 4(\mathrm{n}=9)$ as well as an increased level of ESR $(\mathrm{n}=9)$ or $\operatorname{IgG}(\mathrm{n}=8)$. Table 2 presented the case series of 41 cases with confirmed autoimmune disease-related CCSVI in detail.

There was a significant difference between CCSVI with or without immunological etiology in terms of symptoms and signs (Table 1). CCSVI patients with suspected/ subclinical autoimmune disease presented at an older age on admission $(\mathrm{P}=0.017)$ and had a higher prevalence of head noises $(\mathrm{P}=0.045)$, while those with confirmed autoimmune disease were more prone to have papilledema $(\mathrm{P}=0.004)$. Intriguingly, the groups with immunological etiology did not show a higher incidence of thrombophilia or increased proinflammatory factors (e.g., neutrophil, IL-6) as we previously expected than the group with non-immunological etiology. However, patients with non-immunological etiology had a higher baseline level of CRP $(\mathrm{P}=0.015)$, which further predicted that an independent inflammatory process might involve in the pathogenesis of CCSVI.

\section{Subgroup analysis in CCSVI with immunological etiology}

We further conducted a subgroup analysis between CCSVI with confirmed autoimmune diseases and suspected/ subclinical autoimmune diseases, particularly focusing on the difference of inflammatory biomarkers between these two subgroups (Table 3). CCSVI patients with APS and IgG4-RD had a relatively younger age than that with SS and a higher CRP level than that with decreased C3. The gender difference was also remarkable. The group of CCSVI with confirmed autoimmune diseases was female-dominated while that of CCSVI with suspected autoimmune diseases (e.g., group of increased ESR and $\mathrm{IgG}$ ) was male-dominated.

\section{Correlations between inflammatory cells and inflammatory cytokines}

Correlation coefficients were calculated with Spearman's test among age, baseline NLR, PLR, RDW, IL-6, CRP, and NSE in groups with non-immunological etiology (Figure 1A), with suspected/subclinical autoimmune disease (Figure 1B), and with confirmed autoimmune disease (Figure 1C). As shown in Table 4, baseline PLR level was moderately correlated to NLR and CRP in the group of CCSVI patients with non-immunological etiology and suspected/ subclinical autoimmune disease, indicating CCSVI itself may relate to the inflammatory process (Table 4 , a and b). However, the level of IL-6 was only positively associated with CRP and age in the group of CCSVI patients with confirmed autoimmune disease (Table 4, c).

\section{Discussion}

To our knowledge, this is the first study evaluating the comprehensive clinical features of CCSVI with immunological disease background. In this study, a case series with 41 patients with confirmed autoimmune diseaserelated CCSVI was presented. Eight different immunecomplex diseases, including APS, SS, IgG4-RD, BD, autoimmune hepatitis, Wegener's granulomatosis, systemic sclerosis, and AQP4-antibody (AQP4-IgG)-positive neuromyelitis optica spectrum disorder (AQP4 ${ }^{+} \mathrm{NMOSD}$ ) were analyzed. CCSVI cases combined with IgG4-RD, SS, and $\mathrm{AQP} 4^{+} \mathrm{NMOSD}$-related CCSVI were never reported before. Furthermore, we explored the inflammatory and coagulation status in CCSVI patients with immunological etiology.

Groups with immunological etiology (including CCSVI patients with confirmed autoimmune disease and suspected/ subclinical autoimmune disease) did not show a higher incidence of thrombophilia or increased pro-inflammatory factors (e.g., neutrophil, IL-6). However, patients with nonimmunological etiology had a higher baseline level of CRP. Besides, baseline PLR level was moderately correlated to NLR and CRP in CCSVI patients with non-immunological etiology and suspected/subclinical autoimmune disease. Due to these findings, we postulated that an independent inflammatory process might involve in the pathogenesis of CCSVI, facilitated by multiple risk factors, among which autoimmune disease background could play a major role. In the subgroup analysis of CCSVI patients with immunological etiology, patients with confirmed autoimmune disease had a higher prevalence of thyroid dysfunction and HBV infection. Apart from having elevated autoimmune antibodies, they were also correlated with decreased $\mathrm{C} 3$ or $\mathrm{C} 4$ and increased ESR or IgG. These 


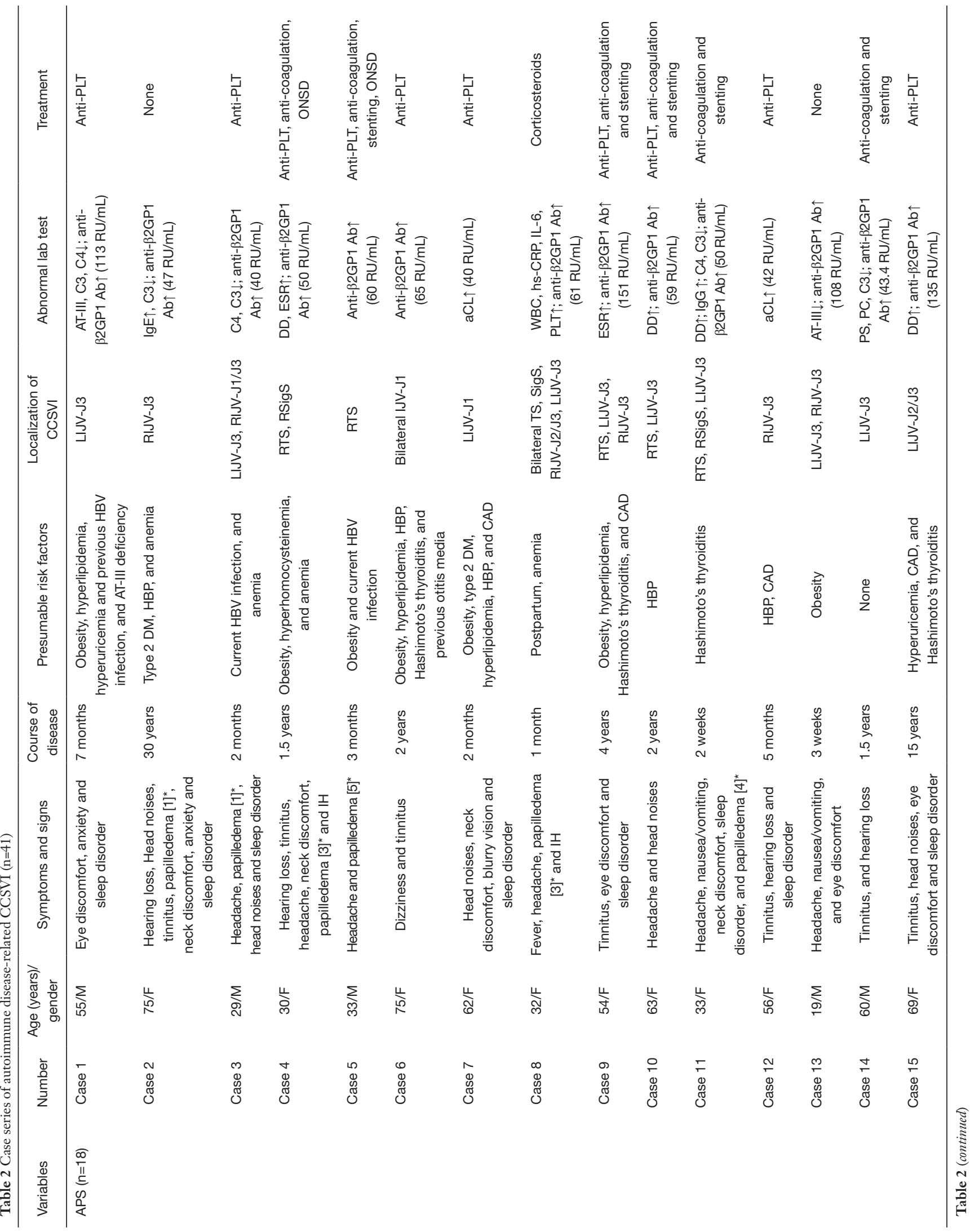




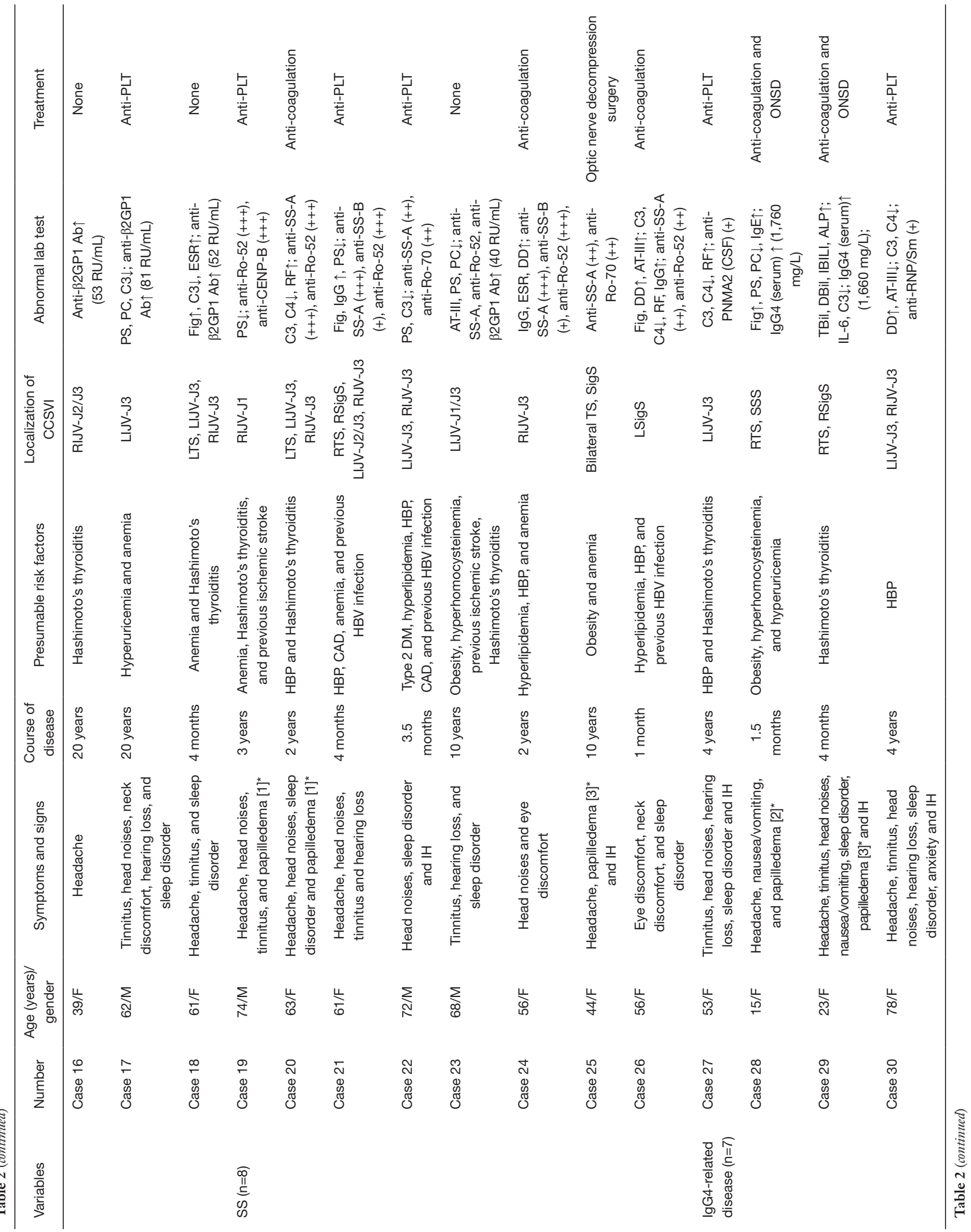




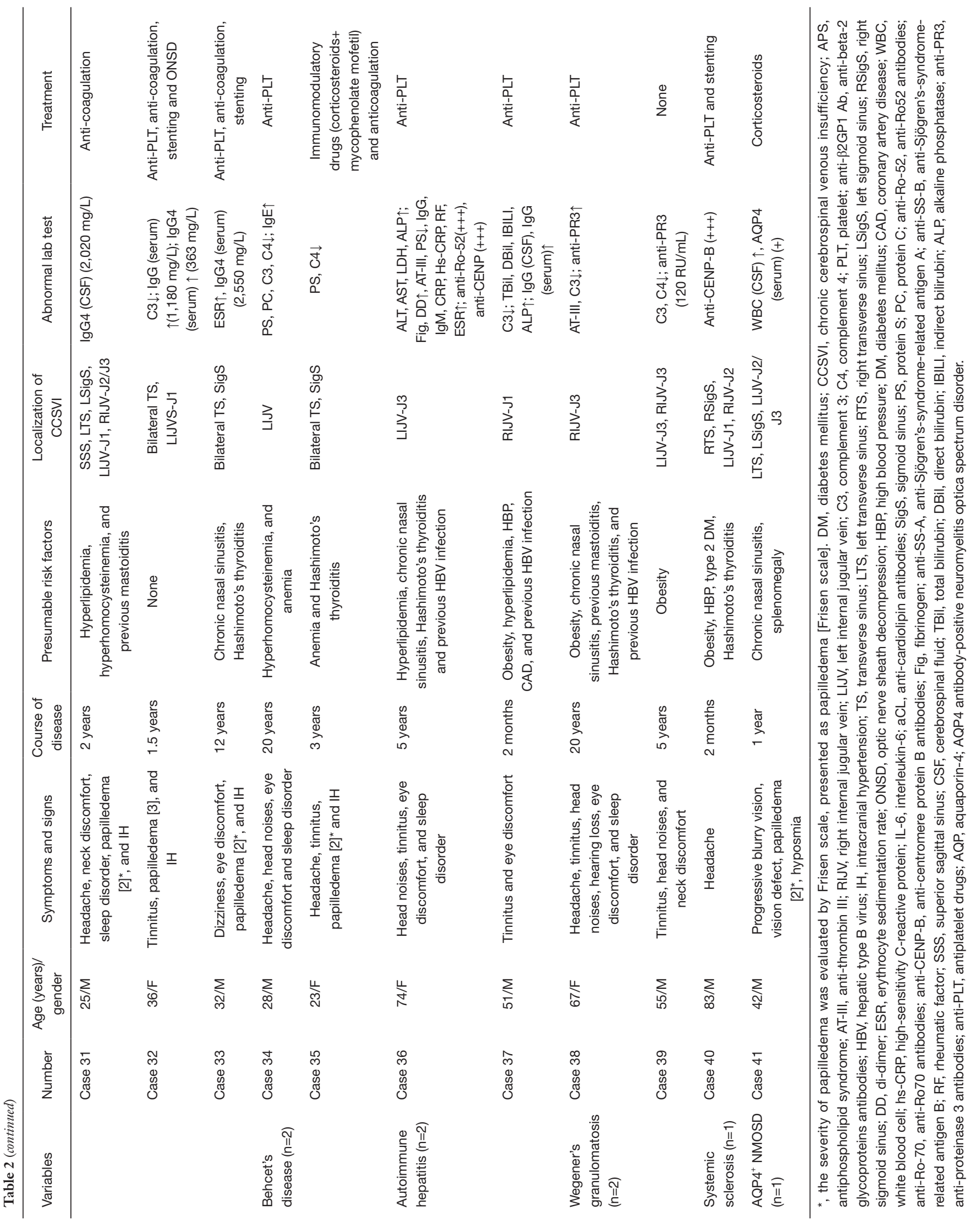


Table 3 Inflammatory biomarkers in subgroup analysis of autoimmune diseases and suspected autoimmune diseases-related CCSVI

\begin{tabular}{|c|c|c|c|c|c|c|c|c|c|c|}
\hline Variables & APS $(n=18)$ & SS $(n=8)$ & $\begin{array}{l}\text { IgG4-related } \\
\text { disease }(n=7)\end{array}$ & $\begin{array}{l}\text { Decreased C3 } \\
\quad(n=76)\end{array}$ & $\begin{array}{c}\text { Decreased C4 } \\
\quad(\mathrm{n}=38)\end{array}$ & $\begin{array}{l}\text { Increased ESR } \\
\quad(n=21)\end{array}$ & $\begin{array}{l}\text { Increased lgG } \\
\qquad(\mathrm{n}=10)\end{array}$ & $\begin{array}{c}G \text { Increased } \lg E \\
(n=5)\end{array}$ & $\begin{array}{c}\text { Positive RF } \\
(n=4)\end{array}$ & $P$ value \\
\hline Age & $49.61 \pm 3.75^{\wedge}$ & $61.75 \pm 3.48$ & $43.00 \pm 9.73^{\wedge}$ & $57.17 \pm 1.49^{\# \wedge}$ & $53.71 \pm 2.75^{\wedge}$ & $58.61 \pm 2.65^{\wedge}$ & $58.90 \pm 5.62$ & $47.00 \pm 6.17^{\wedge}$ & $70.75 \pm 1.75$ & 0.009 \\
\hline $\begin{array}{l}\text { NLR on } \\
\text { admission }\end{array}$ & $1.81 \pm 0.16$ & $2.20 \pm 0.36$ & $1.48 \pm 0.12$ & $1.80 \pm 0.09$ & $1.76 \pm 0.12$ & $1.97 \pm 0.24$ & $1.74 \pm 0.17$ & $1.81 \pm 0.45$ & $1.70 \pm 0.16$ & 0.865 \\
\hline $\begin{array}{l}\text { PLR on } \\
\text { admission }\end{array}$ & $112.13 \pm 9.27$ & $148.78 \pm 16.02$ & $104.96 \pm 13.76$ & $121.59 \pm 5.36$ & $117.43 \pm 7.57$ & $123.45 \pm 6.41$ & $108.23 \pm 8.73$ & $139.56 \pm 25.72$ & $116.68 \pm 8.80$ & 0.472 \\
\hline $\begin{array}{l}\text { PLR at } \\
\text { discharge }{ }^{\&}\end{array}$ & NR & $143.48 \pm 21.89$ & $99.46 \pm 4.44$ & $126.27 \pm 16.43$ & $151.60 \pm 12.82$ & $121.41 \pm 29.79$ & NR & NR & NR & 0.695 \\
\hline IL-6 (pg/mL) & $4.66 \pm 1.26$ & $8.72 \pm 5.42$ & $5.80 \pm 2.13$ & $4.82 \pm 0.90$ & $4.99 \pm 1.67$ & $4.75 \pm 1.02$ & $5.30 \pm 1.29$ & $2.66 \pm 0.66$ & $3.57 \pm 0.81$ & 0.291 \\
\hline CRP (mg/L) & $2.44 \pm 0.22^{\S}$ & $2.29 \pm 0.43$ & $2.46 \pm 0.65^{\S}$ & $1.78 \pm 0.10$ & $1.89 \pm 0.16$ & $4.39 \pm 0.76$ & $3.30 \pm 0.85^{\S}$ & $2.66 \pm 0.69$ & $2.08 \pm 0.33$ & $<0.001$ \\
\hline NSE (ng/mL) & $12.70 \pm 0.47$ & $14.75 \pm 1.58$ & $14.97 \pm 2.35$ & $12.57 \pm 0.79$ & $11.58 \pm 0.61$ & $12.82 \pm 0.37$ & $11.58 \pm 0.61$ & $12.57 \pm 0.79$ & $13.39 \pm 1.01$ & 0.536 \\
\hline
\end{tabular}

\&, the number of patients who had complete blood count (CBC) test at discharge $(n=36) ;{ }^{*}$, compared with group of APS, statistically significant at $\mathrm{P}<0.05 ; \wedge$, compared with group of $\mathrm{SS}$, statistically significant at $\mathrm{P}<0.05$; ${ }^{\S}$, compared with group of decreased $\mathrm{C} 3$, statistically significant at $\mathrm{P}<0.05$. CCSVI, chronic cerebrospinal venous insufficiency; APS, antiphospholipid syndrome; SS, Sjögren's syndrome; C3, complement 3; C4, complement 4; ESR, erythrocyte sedimentation rate; RF, rheumatic factor; NLR, neutrophil to lymphocyte ratio; PLR, platelet to lymphocyte ratio; RDW, red blood cell distribution width; IL-6, interleukin-6; CRP, C-reactive protein; NSE, neuron-specific enolase.

A

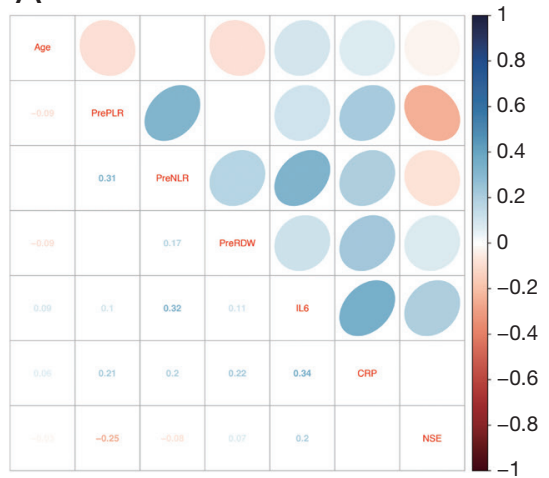

B

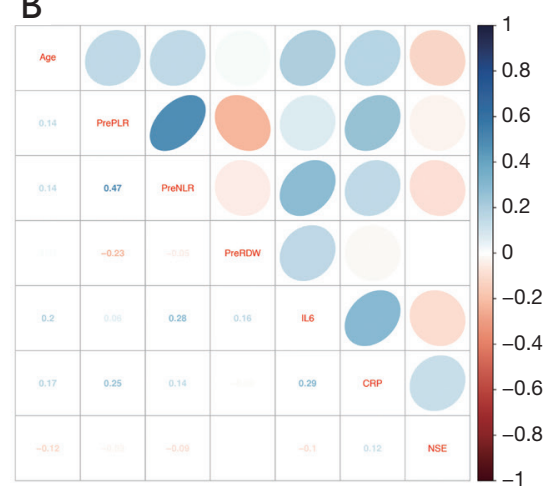

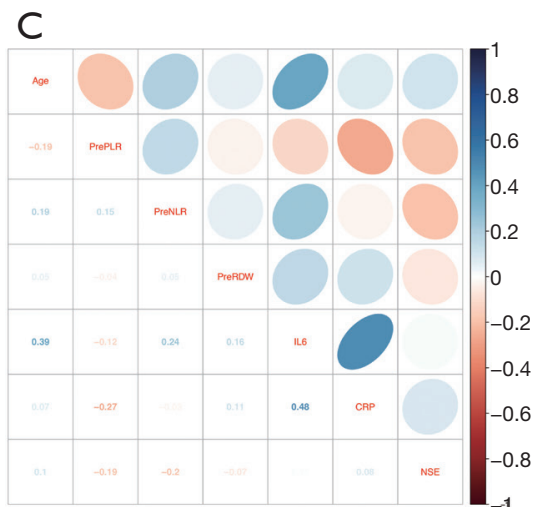

Figure 1 Spearman's correlations between age and inflammatory biomarkers in CCSVI with non-immunological etiology (A), with suspected/subclinical autoimmune disease (B), and with confirmed autoimmune disease (C). CCSVI, chronic cerebrospinal venous insufficiency.

results were consistent with former clinical studies (19-21). Moreover, groups with APS and IgG4-RD presented in a relatively younger population than that with SS and had a higher CRP level than that with decreased C3.
The intricate relationship between inflammation (adaptive immune system) and complement pathway (innate immune system) and hemostasis (coagulation and thrombolysis) in immune-complex-mediated autoimmune 
Table 4 Spearman correlations among inflammatory markers and age

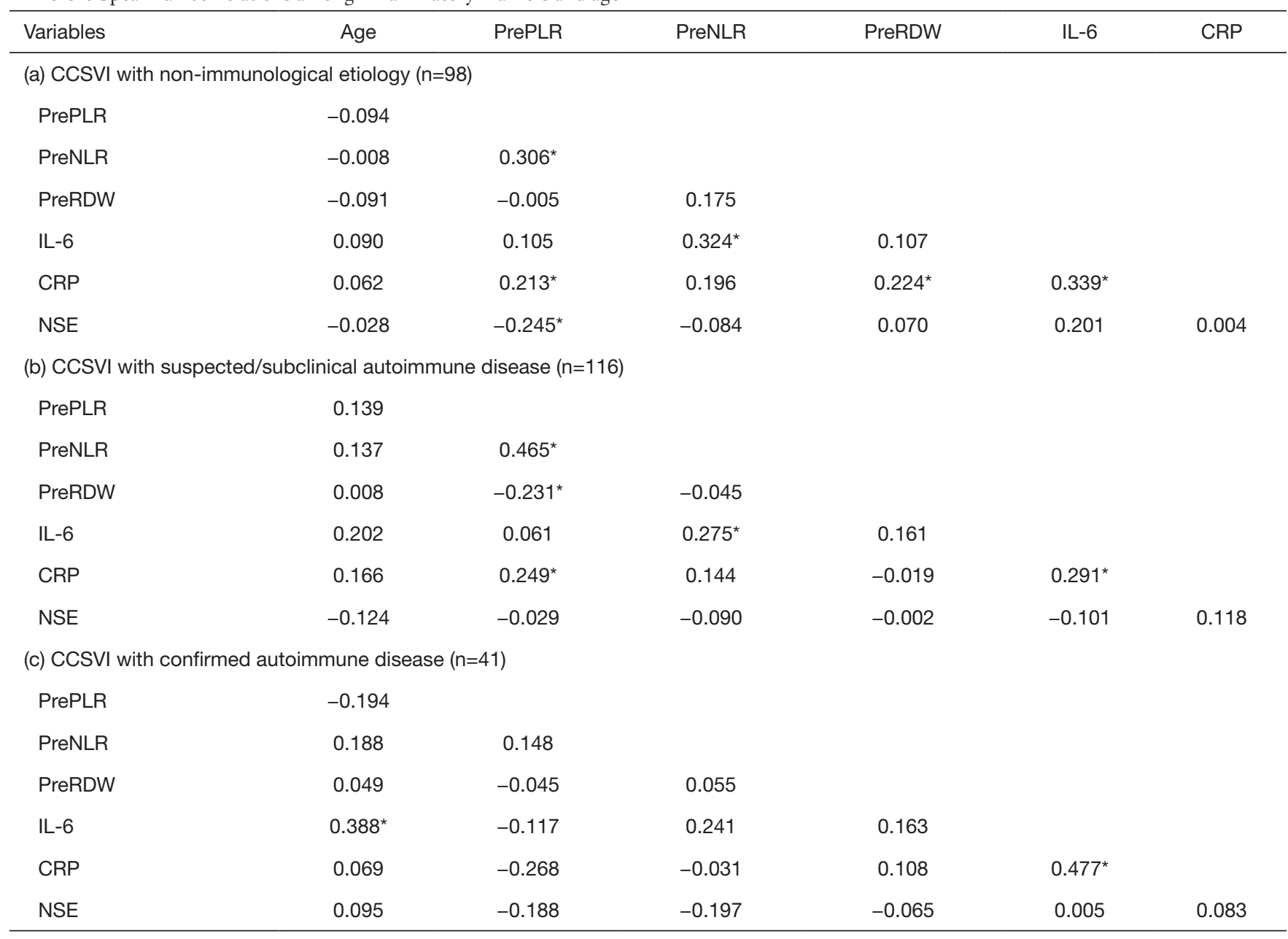

*, statistically significant at $\mathrm{P}<0.05$. PreNLR, neutrophil to lymphocyte ratio on admission; PrePLR, platelet to lymphocyte ratio on admission; PreRDW, red blood cell distribution width on admission; CRP, C-reactive protein; NSE, neuron-specific enolase; IL-6, interleukin-6.

diseases was reviewed in several studies (22-24). Based on their common points, we preferred to explain the mechanism from two perspectives: on a physiological level, the coexistence of hemostatic and inflammatory mediators is served as the first line to protect the body from self-antigens and non-self antigens, also termed as "immunothrombosis" or "thromboinflammation" in recent years $(22,24)$. The common structural characteristic of consisting serine proteinases with trypsin-like activity contributes to the precise interplay between the complement system, the coagulation, and fibrinolytic cascade (25). Certain coagulation factors (FXa, thrombin, plasmin) have $\mathrm{C} 3$ and $\mathrm{C} 5$ convertase activity, contributing to an additional pathway of complement activation $(21,26)$. Complement-derived inflammatory mediators (anaphylatoxin), such as $\mathrm{C} 3 \mathrm{a}, \mathrm{C} 4 \mathrm{a}$, and $\mathrm{C} 5 \mathrm{a}$, could increase vascular permeability, activate neutrophils, promote the release of tumor necrosis factor (TNF) from monocyte, upregulate tissue factor (TF), then initiating extrinsic coagulation pathway (27). Platelet can also be activated by the deposition of C4d split fragments, resulting in the facilitation of the coagulation process (20). While on the pathological level, hypocomplementemia is frequently prevalent in patients with APS and SLE, which predominantly relates to the chronic inflammatory process with the basis of the pathophysiology of immune complexmediated diseases. The overly activated immune system causes the production of self-antigens with unbalanced consumption of complements. With the inverse relationship between complements and their derivatives, anaphylatoxin 
(C3a, C4a, and C5a) would further cause hypercoagulation state and even thrombotic events. Meanwhile, complement regulatory factors also decrease due to either attack from autoimmune antibodies, or increased consumption or lower expression of relevant genes $(28,29)$. For example, during the pathogenesis of APS, beta-2 glycoprotein-I ( $\beta 2$ GPI) undergoes a conformational change from a circular form to an elongated form that can bind C3; then, C3 exposes its binding sites, which is more susceptible to degradation by complement factor $\mathrm{H}(\mathrm{CFH})$ and factor I (30). Moreover, $\beta 2$-GPI shares structural similarity to $\mathrm{CFH}$ so that antibodies could also combine with $\mathrm{CFH}$ (31). With the growing appreciation of complement activation and thrombosis in immune-complex-mediated autoimmune diseases, novel therapies would be fostered, including antiplatelet, anticoagulants as immunomodulators, and targeted molecular therapy toward complements $(25,26)$.

There are several limitations in our study. This is a real-world case-control study of patients with welldefined CCSVI. Patients with a history of autoimmune diseases usually underwent long-term standardized immunomodulation treatment prior to their enrollment, so that the inflammatory biomarker assay tended to be normal despite positive findings of autoimmune antibodies. Therefore, further studies on acute thrombotic events of autoimmune disease are needed. Moreover, our findings indicated the difference in the inflammatory activity among immunological diseases. Based on the complementary relationship between inflammation and thrombosis, we further raised a question on whether the severity of immunological diseases was correlated with increased inflammatory activity and elevated risk of thrombotic events.

\section{Conclusions}

Groups with immunological etiology did not show a higher incidence of thrombophilia or increased pro-inflammatory biomarkers. However, patients with non-immunological etiology had a higher baseline level of CRP. Besides, baseline PLR was moderately correlated to NLR and CRP in CCSVI patients with non-immunological etiology and suspected/subclinical autoimmune disease. Therefore, an independent inflammatory process may involve in the pathogenesis of CCSVI. For those with immunological etiology, autoimmune antibodies-mediated vessel wall damage and hypercoagulation state may also play a major role.

\section{Acknowledgments}

We would like to thank Prof. Yuchuan Ding for his help in polishing the language of our paper.

Funding: This study was sponsored by the National Key R\&D Program of China (2017YFC1308400), the National Natural Science Foundation (81371289), and the Project of Beijing Municipal Top Talent for Healthy Work of China (2014-2-015).

\section{Footnote}

Reporting Checklist: The authors have completed the STROBE reporting checklist. Available at http://dx.doi. org/10.21037/atm-20-4201

Data Sharing Statement: Available at http://dx.doi. org/10.21037/atm-20-4201

Conflicts of Interest: All authors have completed the ICMJE uniform disclosure form (available at http://dx.doi. org/10.21037/atm-20-4201). The authors have no conflicts of interest to declare.

Ethical Statement: The authors are accountable for all aspects of the work in ensuring that questions related to the accuracy or integrity of any part of the work are appropriately investigated and resolved. The study was conducted in accordance with the Declaration of Helsinki (as revised in 2013). The study was approved by Ethics Committee of Xuanwu Hospital, Capital Medical University (2019-006), and informed consent was taken from all individual participants.

Open Access Statement: This is an Open Access article distributed in accordance with the Creative Commons Attribution-NonCommercial-NoDerivs 4.0 International License (CC BY-NC-ND 4.0), which permits the noncommercial replication and distribution of the article with the strict proviso that no changes or edits are made and the original work is properly cited (including links to both the formal publication through the relevant DOI and the license). See: https://creativecommons.org/licenses/by-nc-nd/4.0/.

\section{References}

1. Albayram S, Kantarci F. Prevalence, sensitivity, and specificity of chronic cerebrospinal venous insufficiency in 
MS. Neurology 2011;77:e126.

2. Simka M, Kazibudzki M. Chronic cerebrospinal venous insufficiency and multiple sclerosis: A commentary. Ann Neurol 2010;68:562-3; author reply 563-4.

3. Zivadinov R, Ramanathan M, Dolic K, et al. Chronic cerebrospinal venous insufficiency in multiple sclerosis: diagnostic, pathogenetic, clinical and treatment perspectives. Expert Rev Neurother 2011;11:1277-94.

4. Laupacis A, Lillie E, Dueck A, et al. Association between chronic cerebrospinal venous insufficiency and multiple sclerosis: a meta-analysis. CMAJ 2011;183:E1203-E1212.

5. Traboulsee AL, Knox KB, Machan L, et al. Prevalence of extracranial venous narrowing on catheter venography in people with multiple sclerosis, their siblings, and unrelated healthy controls: a blinded, case-control study. Lancet 2014;383:138-45.

6. Sundstrom P, Wahlin A, Ambarki K, et al. Venous and cerebrospinal fluid flow in multiple sclerosis: a case-control study. Ann Neurol 2010;68:255-9.

7. Zivadinov R, Marr K, Cutter G, et al. Prevalence, sensitivity, and specificity of chronic cerebrospinal venous insufficiency in MS. Neurology 2011;77:138-44.

8. Singh RK, Bhoi SK, Kalita J, et al. Cerebral Venous Sinus Thrombosis Presenting Feature of Systemic Lupus Erythematosus. J Stroke Cerebrovasc Dis 2017;26:518-22.

9. Steinlin MI, Blaser SI, Gilday DL, et al. Neurologic manifestations of pediatric systemic lupus erythematosus. Pediatr Neurol 1995;13:191-7.

10. Wang L, Chen H, Zhang Y, et al. Clinical Characteristics of Cerebral Venous Sinus Thrombosis in Patients with Systemic Lupus Erythematosus: A Single-Centre Experience in China. J Immunol Res 2015;2015:540738.

11. Duman T, Demirci S, Uluduz D, et al. Cerebral Venous Sinus Thrombosis as a Rare Complication of Systemic Lupus Erythematosus: Subgroup Analysis of the VENOST Study. J Stroke Cerebrovasc Dis 2019;28:104372.

12. Shi J, Huang X, Li G, et al. Cerebral venous sinus thrombosis in Behcet's disease: a retrospective case-control study. Clin Rheumatol 2018;37:51-7.

13. Sorgun MH, Rzayev S, Kural MA, et al. Cerebral Venous Thrombosis in Behcet's Disease Patients Compared to Other Causes of Cerebral Venous Thrombosis: a Retrospective Study. Arch Rheumatol 2016;31:248-53.

14. Shlebak A. Antiphospholipid syndrome presenting as cerebral venous sinus thrombosis: a case series and a review. J Clin Pathol 2016;69:337-43.

15. Sareen D, Jain A, Paljor P. Pseudotumor syndrome associated with antiphospholipid antibodies and cerebral venous sinus thrombosis. J Assoc Physicians India 2002;50:603-5.

16. Standridge $\mathrm{S}$, de los Reyes E. Inflammatory bowel disease and cerebrovascular arterial and venous thromboembolic events in 4 pediatric patients: a case series and review of the literature. J Child Neurol 2008;23:59-66.

17. Gilyarov MY, Belikova LP, Shchukin IA, et al. Neurological disorders in eosinophilic granulomatosis with polyangiitis (Churg-Strauss syndrome). Zh Nevrol Psikhiatr Im S S Korsakova 2016;116:93-102.

18. Shuleshova NV, Skoromets AA, Matskevich OR, et al. Acute cerebral sinus-thrombosis due to polyangiitis overlap syndrome with granulomatosis with polyangiitis (Wegener's granulomatosis) and eosinophilic granulomatosis with polyangiitis (Churg-Strauss syndrome). Zh Nevrol Psikhiatr Im S S Korsakova 2016;116:30-5.

19. Scambi C, Ugolini S, Tonello M, et al. Complement activation in the plasma and placentas of women with different subsets of antiphospholipid syndrome. Am J Reprod Immunol 2019;82:e13185.

20. Petri MA, Conklin J, O'Malley T, et al. Platelet-bound $\mathrm{C} 4 \mathrm{~d}$, low $\mathrm{C} 3$ and lupus anticoagulant associate with thrombosis in SLE. Lupus Sci Med 2019;6:e000318.

21. Foley JH, Walton BL, Aleman MM, et al. Complement Activation in Arterial and Venous Thrombosis is Mediated by Plasmin. EBioMedicine 2016;5:175-82.

22. Gaertner F, Massberg S. Blood coagulation in immunothrombosis-At the frontline of intravascular immunity. Semin Immunol 2016;28:561-9.

23. Engelmann B, Massberg S. Thrombosis as an intravascular effector of innate immunity. Nat Rev Immunol 2013;13:34-45.

24. Jackson SP, Darbousset R, Schoenwaelder SM. Thromboinflammation: challenges of therapeutically targeting coagulation and other host defense mechanisms. Blood 2019;133:906-18.

25. Oikonomopoulou K, Ricklin D, Ward PA, et al. Interactions between coagulation and complement-their role in inflammation. Semin Immunopathol 2012;34:151-65.

26. Keragala CB, Draxler DF, McQuilten ZK, et al. Haemostasis and innate immunity - a complementary relationship: A review of the intricate relationship between coagulation and complement pathways. Br J Haematol 2018;180:782-98.

27. Oku K, Nakamura H, Kono M, et al. Complement and thrombosis in the antiphospholipid syndrome. Autoimmun 
Rev 2016;15:1001-4.

28. Chaturvedi S, Brodsky RA, McCrae KR. Complement in the Pathophysiology of the Antiphospholipid Syndrome. Front Immunol 2019; 10:449.

29. Chaturvedi S, Braunstein EM, Yuan X, et al. Complement activity and complement regulatory gene mutations are associated with thrombosis in APS and CAPS. Blood
2020;135:239-51.

30. Gropp K, Weber N, Reuter M, et al. beta(2)-glycoprotein I, the major target in antiphospholipid syndrome, is a special human complement regulator. Blood 2011;118:2774-83.

31. Ferluga J, Kishore U, Sim RB. A potential anticoagulant role of complement factor H. Mol Immunol 2014;59:188-93.
Cite this article as: Song SY, Lan D, Wu XQ, Ding YC, Ji XM, Meng R. Clinical characteristics, inflammation and coagulation status in patients with immunological diseaserelated chronic cerebrospinal venous insufficiency. Ann Transl Med 2021;9(3):236. doi: 10.21037/atm-20-4201 
Supplementary

Table S1 Detailed information on coagulation and inflammatory kits and instruments

\begin{tabular}{lr}
\hline Product name & Company name \\
\hline CRP (Human) ELISA Kit & R\&D Systems \\
IL-6 ELISA & R\&D Systems \\
Human Enolase 2/Neuron-specific Enolase Quantikine ELISA Kit & R\&D Systems \\
Antinuclear antibodies (ANAs), anti-neutrophil cytoplasmic antibodies (ANCAs), and antiphospholipid antibodies & R\&D Systems \\
(APLAs) ELISA Kit & R\&D Systems \\
C3, C4 ELISA Kit & R\&D Systems \\
Human fibrinogen ELISA Kit & REAADS \\
REAADS Monoclonal Free Protein S & REAADS \\
REAADS Protein C Antigen & Technozym \\
Technozym ${ }^{\circledR}$ D-Dimer ELISA & R\&D Systems \\
Antithrombin-III ELISA Kit &
\end{tabular}

